\title{
Signatures of universal four-body phenomena and their relation to the Efimov effect
}

\section{J. von Stecher, J. P. D'Incao and Chris H. Greene ${ }^{\star}$}

The problem of three interacting quantal bodies, in its various guises, seems deceptively simple, but it has also provided striking surprises, such as the Efimov effect ${ }^{1,2}$, which was confirmed experimentally ${ }^{3}$ only more than 35 years after its initial prediction. The importance of understanding the three-body problem was magnified by the explosion of ultracold science following the formation of Bose-Einstein condensates in 1995 (ref. 4). For ultracold gases, three-body recombination (where $B+B+B$ collide to form $B_{2}+B$ ) was quickly recognized as the main loss process and connected ${ }^{5-8}$ with the Efimov effect in the 'universal' realm of very large atom-atom scattering lengths $a$. The problem of four interacting bodies challenges theory far more than the three-body quantal problem. Some key insights have been achieved in recent years ${ }^{9-16}$. Here, we present a major extension of our understanding of the four-body problem in the universal large- $a$ regime. Our results support a previous conjecture $^{10}$ that two resonantly bound four-body states are attached to every universal three-body Efimov resonance and they improve the calculated accuracy of their universal properties. A hitherto unanalysed feature found in ultracoldgas experiments ${ }^{3}$ supports this universal prediction, and it provides the first evidence of four-body recombination (where $B+B+B+B$ form $B_{3}+B_{1} B_{2}+B+B$ or $\left.B_{2}+B_{2}\right)$.

The experiment ${ }^{3}$ that observed strong evidence for the long-predicted Efimov effect ${ }^{1,2}$ has spawned a new level of confidence in our theoretical understanding of the three-body problem with short-range forces. However, even though in some respects the three-body problem is beginning to seem 'almost solved', the next step in complexity-to four interacting particles-remains at a primitive stage, comparatively speaking. Although a few studies have been pursued ${ }^{9-12}$, the non-perturbative four-boson problem is still largely uncharted territory, especially for processes that begin or end with three or four free particles. Our present study relates most to the pioneering work of Hammer, Platter and Meißner ${ }^{9}, 10$, and of Yamashita et al. ${ }^{11}$. It concerns a key question in strongly interacting few-body systems: are universal principles of Efimov physics relevant for the four-boson problem? In the early 1970s, the nuclear physicist Vitaly Efimov ${ }^{1,2}$ predicted on very general grounds that three neutral bosons, whose mutual interaction is characterized by a large value for the two-body $s$-wave scattering length $a$, can form a large number of weakly bound states whenever $|a| \gg r_{0}$, where $r_{0}$ is the characteristic range of the interaction. Surprisingly, this could happen even when none of the pairs can bind $(a<0)$. Here we provide an analysis that convincingly demonstrates the existence of a class of universal four-boson states that are intimately related to the Efimov effect. Our results connect with and extend previous analyses ${ }^{9-11}$ and provide a more complete landscape of the universal four-boson phenomena. In addition, we demonstrate how four-boson universal states can be seen (and in retrospect, apparently have already been seen) in ultracold-gas experiments.

Our theoretical model hinges on the tunable interaction strength achievable in ultracold-gas experiments. For alkali atoms, when an external $B$ field is placed near a Fano-Feshbach resonance ${ }^{17}$ a small change of $B$ can cause $a$ to vary from $-\infty$ to $\infty$, allowing for the exploration of a vast range of interatomic interaction strengths. We mimic such variations in $a$ by explicitly modifying the interatomic interactions ${ }^{5}$. In our framework, the solution of the four-body problem culminates with the solution of the 'hyperradial' Schrödinger equation:

$$
-\frac{\hbar^{2}}{2 m} \frac{\mathrm{d}^{2}}{\mathrm{~d} R^{2}} F_{v}(R)+\sum_{v^{\prime}} W_{v v^{\prime}}(R) F_{v^{\prime}}(R)=E F_{v}(R)
$$

where the hyper-radius $R$ describes the overall size of the system. Here, $m$ is the atomic mass, $E$ the total energy and $F_{v}(R)$ the hyper-radial wavefunction, with $v$ representing the set of quantum numbers needed to label each channel. In the hyperspherical adiabatic representation, ${ }^{5,18}$ most of the complexity of the problem arises in solving the hyperangular equations to determine the effective potentials $W_{v v}(R)$ and couplings $W_{v v^{\prime}}(R)$, in this case using a correlated Gaussian hyperspherical approach ${ }^{19}$. The reduction of the problem to the hyper-radial Schrödinger equation (1) then leads to a simple, intuitive picture: the effective potentials $W_{v v}(R)$ support all bound and quasibound states of the system, and the offdiagonal non-adiabatic couplings $W_{\nu v^{\prime}}(R)$ drive inelastic transitions among different channels.

We explore the universality of the four-boson system and its relation to Efimov physics by solving the Schrödinger equation (1) for different model potentials using two complementary numerical techniques: the adiabatic hyperspherical approximation ${ }^{18,20}$, and the correlated Gaussian basis set expansion ${ }^{14,21}$. We base our conclusions on the analysis of energies, potential curves and wavefunctions that describe ground and excited states (see Supplementary Information for details) in the universal regime $|a| \gg r_{0}$, where $r_{0}$ is the characteristic length scale of the two-body interaction, usually associated with the van der Waals length. Figure 1a shows the 'generalized Efimov plot'10, with all important features that relate two-, three- and four-body physics. Figure 1a shows our numerical results for the four-boson energies (solid black lines) along with the dimer-dimer and dimer-atom-atom break-up thresholds for $a>0$ (solid red lines) and the energies of the Efimov states (dashed green lines) representing the atom-trimer break-up threshold. Figure 1c,d conveys the geometrical nature of the fourbody states taking into account the extremely 'floppy' nature of the Efimov trimer states, in which all possible triangular shapes, and even linear configurations, are comparably probable ${ }^{22,23}$.

Figure 1a implies that the four-boson spectrum, throughout the range $r_{0} /|a| \ll 1$, is characterized by precisely two tetramer states 


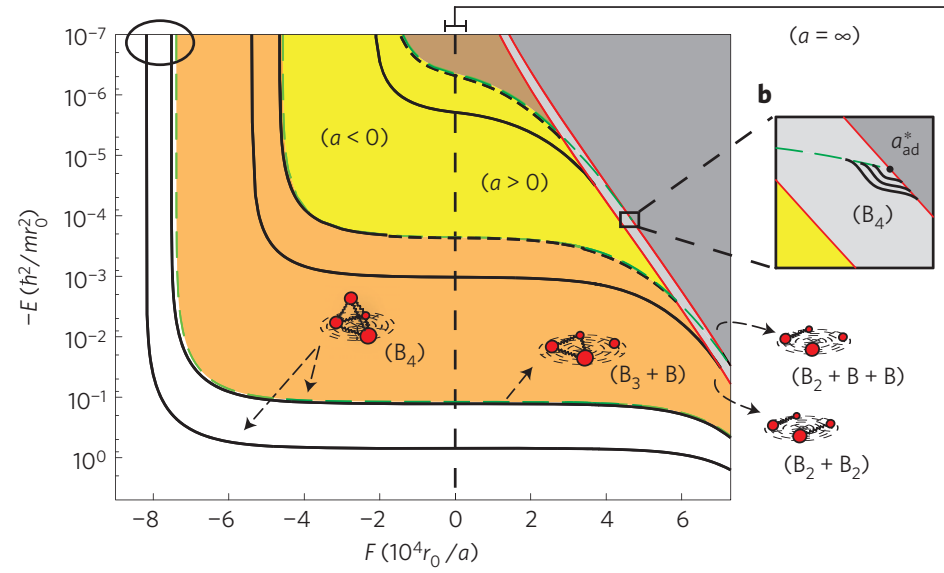

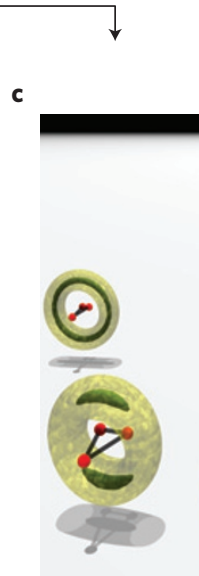

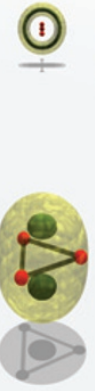

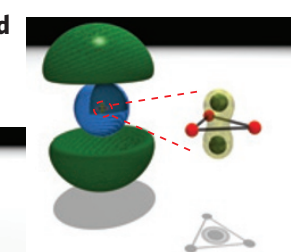

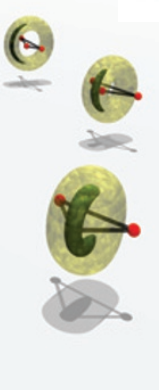

Figure 1 | Spectrum of energies and geometric structure of four-boson states and their connection to the Efimov physics. a,The energy spectrum of four bosons as a function of the atom-atom scattering length a using the auxiliary function $F(x) \equiv \operatorname{sgn}(x) \ln (1+|x|)$ designed to aid the visualization of the full energy landscape. Black solid lines correspond to four-body states, dashed green lines represent atom-trimer dissociation thresholds and red lines correspond to dimer-atom-atom (upper) and dimer-dimer (lower) thresholds. b, Schematic description of dimer-atom-atom Efimov states.

c, d, Geometrical nature of ground and excited four-body states, respectively, for different trimer configurations. For the four-boson ground state, the isosurfaces shown are those where the probability of finding the fourth atom is 0.9 and 0.99 of the maximum probability for that particular trimer geometry. The probability isosurface for the first excited four-boson state in the portion of space where the Efimov trimer resides at its most probable equilateral triangular geometry implies that the fourth atom is very weakly bound, and its size exceeds that of the ground state considerably.

that are associated with each three-body Efimov state, confirming the ref. 10 prediction. In fact, our extensive numerical tests show that these four-boson energies obey a universal relationship to the corresponding Efimov state energy, which at unitarity $(|a|=\infty)$ can be expressed as

$$
E_{4 b}^{(n, m)}=c_{m} E_{3 b}^{(n)}
$$

where $E_{3 b}^{(n)}$ is the energy of the $n$th Efimov state, $n=0,1,2, \ldots$, and $E_{4 b}^{(n, m)}, m=1$ and 2, are the two tetramer energies associated with it (see Supplementary Information for details). Here, we find that the universal relation between three- and four-body energies is characterized by these two universal numbers, equal to: $c_{1} \approx 4.58$ and $c_{2} \sim 1.01$. For the lowest two four-boson states ref. 10 obtained $c_{1} \approx 5$ and $c_{2} \sim 1.01$. Similar values, less deep in the universal regime, can be extracted from the small $B_{2}$ limit of equations (39) and (41) of ref. 9. Our own calculations differ from the universal values if we consider the lowest four-body states (see Supplementary Information). In fact, we believe that the ability of the current method to calculate many more weakly-bound energy levels than previous techniques has been decisive, and it permits us to verify the universal numbers up to $2 \%$ accuracy and resolve a previously-existing disagreement in the literature, between the results of Hammer, Platter and Meißner ${ }^{9,10}$ and those of Yamashita et al. ${ }^{11}$.

The resolution of this controversy is related to the fundamental question of whether or not an additional 'four-body parameter', encapsulating non-universal aspects from the details of the interactions, is required to specify the nature of the four-boson spectrum and scattering observables, akin to the usual three-body parameter ${ }^{1,2,5-7,24,25}$. Specifically, we support the conclusions of refs $9,10,12$ that no additional four-body parameter enters at leading order in the description of universal four-body spectra and scattering properties. Our result also explains the observations of Yamashita et al. ${ }^{11}$ that the energies of more compact fourboson states can vary depending on the details of the interatomic interactions. Our understanding emerges from Fig. 2, showing the four-body effective potentials calculated at unitarity, $|a|=\infty$. We

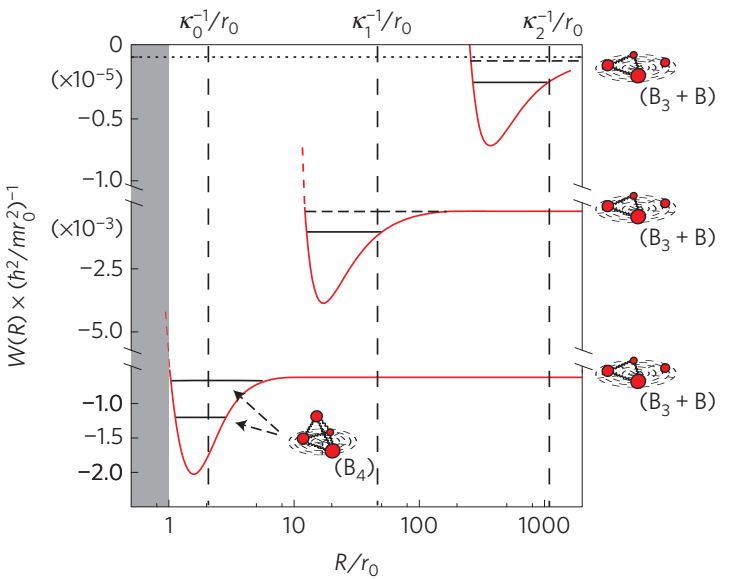

Figure 2 | Effective four-boson potentials for $|a|=\infty$ converging at large $\boldsymbol{R}$ to the atom-trimer thresholds. Solid and dashed black lines represent the four-boson states shown in Fig. 1a. The position in $R$ of the minimum of these potentials scales with the size of the Efimov state, indicated in the figure by $\kappa_{i}^{-1}$ (see Supplementary Information for definition). Therefore, when the trimer state is large the four-boson states associated with it will lie at large $R$, preventing access to the non-universal region $R \lesssim r_{0}$, whereby the four-boson states are universal.

have verified that the effective-potential curves scale with the size of the trimer state. Therefore, if the lowest Efimov state has a size that exceeds $r_{0}$ only marginally, the minimum of the four-body potential is close enough to $r_{0}$ and its four-body states are affected by the shape of the two-body interaction, that is, non-universal physics. On the other hand, if the lowest Efimov state is large compared with $r_{0}$, then the minimum of the four-body potential lies at $R \gg r_{0}$ and the four-boson states probe almost no non-universal effects. Furthermore, the scaling behaviour of the potential curves implies that the three-body Efimov effect controls the four-boson spectrum. As a consequence, the four-boson states follow the same geometric scaling as the three-boson states, with successive energies related by the factor $\mathrm{e}^{-2 \pi / s_{0}} \approx 1 / 515$ and successive radii expanding by the 


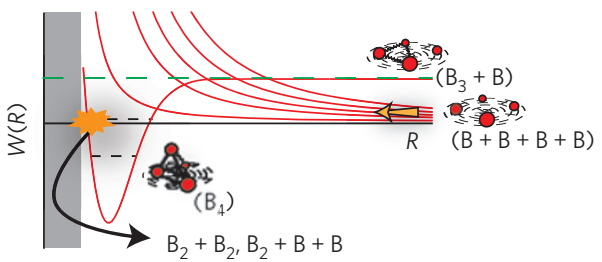

c

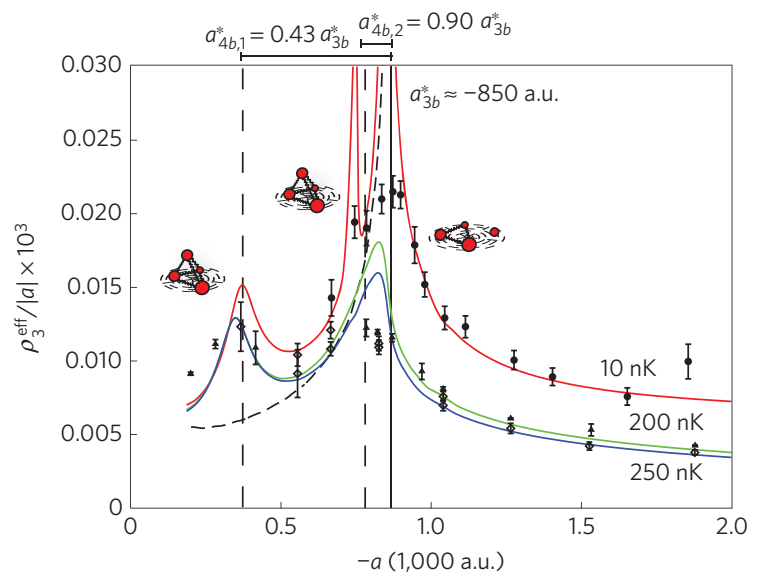

b

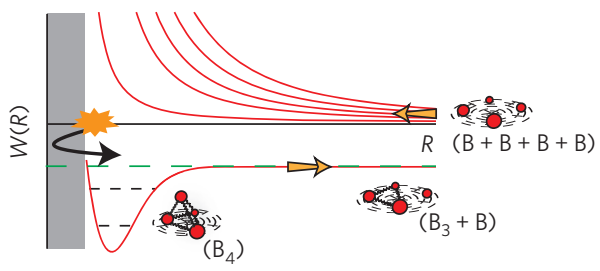

d

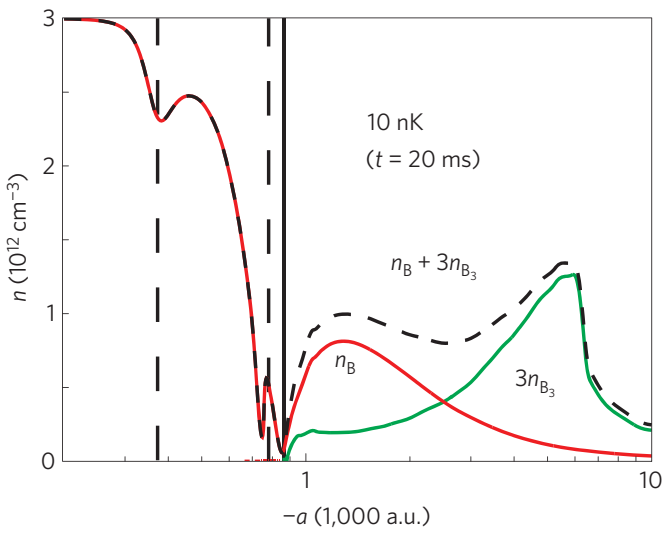

Figure 3 | Evidence of signatures of four-boson states through four-body recombination. $\mathbf{a}$, $\mathbf{b}$, Schematic representation of the pathways for four-body recombination when the Efimov state (green dashed line) is above and below the four-body break-up threshold, respectively. In a the only possible decay channels are associated with deeply bound (two- and three-body) states, whereas in $\mathbf{b}$ the main decay channel is to the available Efimov state plus a free atom. When a four-boson state crosses the four-body dissociation threshold, it resonantly affects the four-body recombination rate, enhancing the rate of atom losses in an ultracold gas. c, Comparison of $\rho_{3}^{\text {eff }}$ (equation (4)) with the experimental data from ref. 3. The shown error bars refer to statistical uncertainty only (see ref. 3 for details). The vertical lines identify the critical scattering lengths where an Efimov state and its associated four-body states

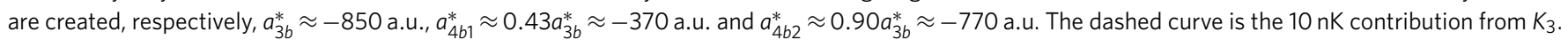
d, The atom and trimer densities at $10 \mathrm{nK}$, demonstrating that four-body recombination can be used to form Efimov trimers.

factor $\mathrm{e}^{\pi / s_{0}} \approx 22.7$ (where $s_{0} \approx 1.00624$ ). These four-boson states are not true bound states, of course, as was pointed out in ref. 10, so the preceding discussion relates to the real part of their energies.

Parenthetically, we also confirm the existence of a class of four-body states that represent the Efimov effect for three bodies. As pointed out in ref. 8, these four-atom states occur whenever an Efimov state is created for $a>0$ (see Fig. 1b). In this case the atom-dimer scattering length, $a_{\mathrm{ad}}$, has a pole and whenever $\left|a_{\mathrm{ad}}\right| \gg a$ a series of four-atom, three-body Efimov states, namely dimer-atom-atom states. Numerically, we observe the emergence of an attractive dipole potential $\left(\propto-1 / R^{2}\right)$ in the dimer-atom-atom channel, for $a \ll R \ll\left|a_{\mathrm{ad}}\right|$, confirming the existence of such four-boson states. In fact, the recent observation of an Efimov resonance in ref. 26 in an ultracold atom-molecule mixture could enable the probing of such four-body states experimentally. We also confirm the non-existence of a 'true Efimov effect' for four bosons in the spirit of the ref. 27 prediction (see Supplementary Information).

The universal four-body physics discussed above can readily be observed in ultracold quantum gases. In general, weakly bound states deeply affect the collisional properties of ultracold gases, enhancing the atomic and/or molecular losses. The relative importance of four-body processes, however, remains largely unexplored, and we could argue that such processes should be far less likely than two-body or three-body collisions in a typical lowdensity gas. On the other hand, near the threshold for formation of any four-boson states, the four-body scattering observables should show a resonant enhancement that dramatically affects the collisional behaviour of the gas, even at low densities. The results of the Innsbruck experiment ${ }^{3}$, realized at atomic densities of $n(0) \approx 3 \times 10^{12} \mathrm{~cm}^{-3}$, were interpreted under the premise that the atom loss stems just from three-body recombination,
$\mathrm{B}+\mathrm{B}+\mathrm{B} \rightarrow \mathrm{B}_{2}+\mathrm{B}+\mathrm{E}_{\text {rel. }}$, which releases enough kinetic energy to eject the collision products. Sure enough, the experimental data show a resonant peak in the three-body recombination rate $K_{3}$, more specifically at a three-body recombination length of $\rho_{3}=\left[2 m K_{3} /(\sqrt{3} \hbar)\right]^{1 / 4}$, at $a=-850$ a.u., in agreement with theoretical expectations for the manifestation of Efimov physics through three-body recombination ${ }^{5-7}$.

Although the experimental Innsbruck data $^{3}$ are reasonably well understood $^{24,28-30}$, distinguishing three- and four-body losses is difficult, and four-body processes could still be embedded in the observed decay rates. Accordingly, we have reanalysed the Innsbruck data ${ }^{3}$, looking for possible signatures of new four-boson states. The key observation from our results is that for $a<0$, when an Efimov state is created, say at $a=a_{3 b}^{*}$, it is accompanied by the creation of two four-boson states at slightly less negative values of $a$, and those states should enhance four-body processes in this experimentally explored region of $a$. Moreover, our calculations indicate that, once the scattering length $a_{3 b}^{*}$ is known, we know the scattering lengths at which such four-body states appear. This universal relation is determined by the energy spectrum (see Fig. 1a), namely

$$
a_{4 b 1}^{*} \approx 0.43 a_{3 b}^{*}, \quad \text { and } \quad a_{4 b 2}^{*} \approx 0.90 a_{3 b}^{*}
$$

From our numerical calculations we have found that these relations are approximately fulfilled even when $a_{3 b}^{*}$ is not deep in the universal regime, suggesting an insensitivity to non-universal effects.

The main process where such states should appear is four-body recombination, where the four initially free atoms collide to recombine into the dimer-dimer channel, the dimer-atom-atom channel and/or the atom-trimer channel. Figure 3a,b, respectively, 
depicts this process through the effective potentials at scattering lengths very close to the threshold $a_{3 b}^{*}$ for formation of an Efimov state (green dashed line) and just past the point $\left(|a|<\left|a_{3 b}^{*}\right|\right)$ of its creation. When a four-boson state resides energetically close to the collision threshold, we expect a resonant enhancement to the four-body recombination rate, $K_{4}$. A straightforward Wigner threshold-law analysis demonstrates that $K_{4}$ approaches a constant as the collision energy is tuned to zero ${ }^{16,31}$, and thus four-body recombination can indeed potentially compete with three-body recombination in causing atomic losses.

To assess the importance of $K_{4}$ and quantify our predictions, we have calculated $K_{4}$ by numerically solving equation (1) using a formula for $K_{4}$ derived elsewhere ${ }^{31}$. The main difficulty in comparing our results with data is that existing experiments are probably unable to distinguish three- and four-body losses. We therefore introduce an effective three-body recombination rate, in which both three- and four-body physics are included:

$$
K_{3}^{\mathrm{eff}}(a, t)=K_{3}(a)+n(t) K_{4}(a) / 3
$$

where $n(t)$ is the peak atomic density in the trap at time $t$, calculated by solving the time evolution rate equations. Figure $3 \mathrm{c}$ shows our recombination length $\rho_{3}^{\text {eff }}=\left[2 m K_{3}^{\text {eff }} /(\sqrt{3} \hbar)\right]^{1 / 4}$ for $t=20 \mathrm{~ms}$. For $K_{3}$ we use the thermally averaged results of ref. 24 calculated for temperatures of 10,200 and $250 \mathrm{nK}$, and adjust it to fit the Efimov resonance at $a=a_{3 b}^{*}=-850$ a.u. and the experimental data for $|a|>\left|a_{3 b}^{*}\right|$. Our results show that for this range of $a$ three-body recombination is indeed the dominant loss process. For $|a|<\left|a_{3 b}^{*}\right|$, however, we find much better agreement by assuming that four-body recombination is the dominant loss process-the dashed curve in Fig. $3 \mathrm{c}$ is the $10 \mathrm{nK}$ contribution from $K_{3}$. For this range of $a$, as indicated in Fig. $3 c$ by the vertical dashed lines at $a=a_{4 b 1}^{*}$ and $a=a_{4 b 2}^{*}, K_{4}$ is resonantly enhanced when the two four-boson states are created (see the circled region in Fig. 1a). This agreement strongly suggests that the 2006 Innsbruck experiment ${ }^{3}$ also offers the first experimental evidence for the universal fourboson states we discussed here, although the agreement with the second resonance predicted for $a=a_{4 b 2}^{*}$ and $10 \mathrm{nK}$ requires some imagination-and for temperatures of 200 and $250 \mathrm{nK}$ this resonance feature is washed out owing to thermal effects. Nevertheless, the verification of the universal constraint between three- and fourbody physics (equation (3)) strengthens the conclusion that the main resonant feature ${ }^{3}$ at -850 a.u. is indeed an Efimov resonance.

Note also that for $|a|>\left|a_{3 b}^{*}\right|$ four-body recombination to Efimov states, $\mathrm{B}+\mathrm{B}+\mathrm{B}+\mathrm{B} \rightarrow \mathrm{B}_{3}+\mathrm{B}$ (see Fig. $3 \mathrm{~b}$ ), is likely to be the dominant four-body decay pathway. Although three-body recombination tends to dominate the atom loss, the formation of Efimov states through four-body recombination is non-negligible. In Fig. $3 \mathrm{~d}$ we show the atomic density $n_{\mathrm{B}}$ and the density of trimers $n_{\mathrm{B}_{3}}$ at $10 \mathrm{nK}$ for $a$ up to $-10,000$ a.u. Near the threshold for Efimov state formation little energy is released through four-body recombination (approximately the trimer binding energy) and the Efimov state can remain trapped. In this case, our results indicate that about $10 \%$ of the atoms will form trimers. For larger $|a|$, however, the trimer formation is strongly enhanced by a resonance associated with the lowest four-boson state attached to the second Efimov trimer (see Fig. 1a). In this case, we find that about 50\% of the atoms will form trimers. Here, however, the energy released through four-body recombination ejects both the atom and the Efimov trimer. Nevertheless, in an experiment where only atoms are visible, the magnetic field could be set to a value such that $|a|>\left|a_{3 b}^{*}\right|$, the number of atoms measured and the field ramped back to a value such that $|a|<\left|a_{3 b}^{*}\right|$, where no trimers exist. The reappearance of atoms after this ramp would be a convincing signature of the first experimental realization of an ultracold gas of Efimov trimers.
Finally, the Innsbruck group has observed two resonant loss features in four-body recombination ${ }^{32}$ that satisfy our relations in equation (3), offering stronger experimental evidence of the universal four-body physics demonstrated here.

\section{Methods}

Effective three-body recombination. The idea behind the definition of $K_{3}^{\text {eff }}(a, t)$ is to obtain a quantity that encapsulates both three- and four-body contributions Accordingly to our findings, the atomic losses can indeed be seen as an effective three-body recombination within a characteristic timescale, as explained below. That can presumably facilitate a comparison with the available experimental data.

The key ingredient in the derivation of $K_{3}^{\text {eff }}(a, t)$ is the relation between the loss coefficient and the loss rates as given by

$$
L_{\mathrm{N}}=N \frac{1}{N !} K_{\mathrm{N}}
$$

where $N$ is the number of atoms involved in the recombination event. Note that $K_{3}$ (or $K_{4}$ ) represents a fundamental few-body entity, namely, the recombination probability per unit time for a single triad (or tetrad) in a unit volume squared (cubed $)^{17}$. The first factor on the right-hand side of this equation represents the number of atoms lost in the $N$-atom recombination process, whereas the $1 / N$ ! factor accounts for the indistinguishability of the collision partners. The atoms are assumed not to be in a Bose-Einstein condensate.

The rate equation that governs the time evolution of the atomic density can be written as

$$
\frac{\mathrm{d}}{\mathrm{d} t} n(a, t)=-L_{3}(a) n(a, t)^{3}-L_{4}(a) n(a, t)^{4}
$$

or alternatively,

$$
\begin{aligned}
\frac{\mathrm{d}}{\mathrm{d} t} n(a, t) & =-\left[L_{3}(a)+n(a, t) L_{4}(a)\right] n(a, t)^{3} \\
& =-L_{3}^{\text {eff }}(a, t) n(a, t)^{3}
\end{aligned}
$$

from which we define the effective $L_{3}^{\text {eff }}(a, t)$. From this definition, and using the relation between $L_{\mathrm{N}}$ and $K_{\mathrm{N}}$ above, we can easily arrive at our definition of $K_{\mathrm{N}}^{\text {eff }}$ following the steps below:

$$
\begin{gathered}
L_{3}^{\mathrm{eff}}(a, t)=L_{3}(a)+n(a, t) L_{4}(a) \\
3 \frac{L_{3}^{\mathrm{eff}}(a, t)}{3 !}=3 \frac{K_{3}(a)}{3 !}+n(a, t) 4 \frac{K_{4}(a)}{4 !} \\
K_{3}^{\mathrm{eff}}(a, t)=K_{3}(a)+n(a, t) K_{4}(a) / 3
\end{gathered}
$$

We have in fact verified that for times $t<\sim t_{0}=\left[n(0)^{2}\left(K_{3}+n(0) K_{4} / 3\right)\right]^{-1}$ $(\approx 50 \mathrm{~ms}$ for our case) the time dependence of $n(t)$ can be described as a result of the effective three-body rate in equation (4), by setting $n(t)=n(0)$. For longer times, $n(t)$ is affected primarily by three- or four-body processes depending on whether or not $K_{3} \gg n(0) K_{4}$.

Received 29 October 2008; accepted 30 March 2009; published online 26 April 2009

\section{References}

1. Efimov, V. Weakly bound states of three resonantly interacting particles. Yad. Fiz. 12, 1080-1091 (1970); Sov. J. Nucl. Phys. 12, 589-595 (1971).

2. Efimov, V. Energy levels of three resonantly interacting particles. Nucl. Phys. A 210, 157-188 (1973).

3. Kraemer, T. et al. Evidence for Efimov quantum states in an ultracold gas of caesium atoms. Nature 440, 315-318 (2006).

4. Anderson, M. H., Ensher, J. R., Matthews, M. R., Wieman, C. E. \& Cornell, E. A. Observation of Bose-Einstein condensation in a dilute atomic vapor. Science 269, 198-201 (1995).

5. Esry, B. D., Greene, C. H. \& Burke, J. P. Jr. Recombination of three atoms in the ultracold limit. Phys. Rev. Lett. 83, 1751-1754 (1999).

6. Nielsen, E. \& Macek, J. H. Low-energy recombination of identical bosons by three-body collisions. Phys. Rev. Lett. 83, 1566-1569 (1999).

7. Bedaque, P. F., Braaten, E. \& Hammer, H.-W. Three-body recombination in Bose gases with large scattering length. Phys. Rev. Lett. 85, 908-911 (2000).

8. Braaten, E. \& Hammer, H. W. Universality in few-body systems with large scattering length. Phys. Rep. 428, 259-390 (2006). 
9. Platter, L., Hammer, H. \& Meißner, U. Four-boson system with short-range interactions. Phys. Rev. A 70, 52101 (2004).

10. Hammer, H. W. \& Platter, L. Universal properties of the four-body system with large scattering length. Eur. Phys. J. A 32, 113-120 (2007).

11. Yamashita, M. T., Tomio, L., Delfino, A. \& Frederico, T. Four-boson scale near a Feshbach resonance. Europhys. Lett. 75, 555-561 (2006).

12. Hanna, G. J. \& Blume, D. Energetics and structural properties of three-dimensional bosonic clusters near threshold. Phys. Rev. A 74, 063604 (2006).

13. Petrov, D. S., Salomon, C. \& Shlyapnikov, G. V. Weakly bound dimers of fermionic atoms. Phys. Rev. Lett. 93, 090404 (2004).

14. von Stecher, J. \& Greene, C. H. Spectrum and dynamics of the BCS-BEC crossover from a few-body perspective. Phys. Rev. Lett. 99, 090402 (2007).

15. D'Incao, J. P., Rittenhouse, S. T., Mehta, N. P. \& Greene, C. H. Dimer-dimer collisions at finite energies in two-component Fermi gases. Phys. Rev. A 79, 030501 (2009).

16. Wang, Y. \& Esry, B. D. Efimov trimer formation via ultracold four-body recombination. Phys. Rev. Lett. 102, 133201 (2009).

17. Köhler, T., Góral, K. \& Julienne, P. S. Production of cold molecules via magnetically tunable Feshbach resonances. Rev. Mod. Phys. 78, 1311-1362 (2006).

18. Macek, J. H. Properties of autoionizing states of He. J. Phys. B 1, 831-843 (1968).

19. von Stecher, J. Trapped Ultracold Atoms With Tunable Interactions. PhD thesis, Univ. of Colorado, Boulder (2008); $<$ http://jilawww.colorado.edu/pubs/thesis/vonstecher/>.

20. Coelho, H. T. \& Hornos, J. E. Proof of basic inequalities in the hyperspherical formalism for the N-body problem. Phys. Rev. A 43, 6379-6381 (1991).

21. Suzuki, Y. \& Varga, K. Stochastic Variational Approach to Quantum-Mechanical Few-Body Problems. (Springer, 1998).

22. D'Incao, J. P. \& Esry, B. D. Manifestations of the Efimov effect for three identical bosons. Phys. Rev. A 72, 032710 (2005).

23. Esry, B. D. \& Greene, C. H. Quantum physics: A ménage à trois laid bare. Nature 440, 289-290 (2006).

24. D'Incao, J. P., Greene, C. H. \& Esry, B. D. The short-range three-body phase and other issues impacting the observation of Efimov physics in ultracold quantum gases. J. Phys. B 42, 044016 (2009).
25. Danilov, G. S. On the three-body problem in the case of short-range forces. Zh. Eksp. Teor. Fiz. 40, 498-507 (1961); Sov. Phys. JETP 13, 349-355 (1961).

26. Knoop, S. et al. Observation of an Efimov-like trimer resonance in ultracold atom-dimer scattering. Nature Phys. 5, 227-230 (2009).

27. Amado, R. D. \& Greenwood, F. C. There is no Efimov effect for four or more particles. Phys. Rev. D 7, 2517-2519 (1973).

28. Jonsell, S. Efimov states for systems with negative scattering lengths. Europhys. Lett. 76, 8-14 (2006).

29. Lee, M. D., Koehler, T. \& Julienne, P. S. Excited Thomas-Efimov levels in ultracold gases. Phys. Rev. A 76, 012720 (2007).

30. Massignan, P. \& Stoof, H. T. C. Efimov states near a Feshbach resonance. Phys. Rev. A 78, 030701 (2008)

31. Mehta, N. P., Rittenhouse, S. T., D'Incao, J. P., von Stecher, J. \& Greene, C. H. A general theoretical description of N-body recombination. Preprint at $<$ http://arxiv.org/abs/0903.4145> (2009).

32. Ferlaino, F. et al. Evidence for universal four-body states tied to an Efimov trimer. Phys. Rev. Lett. 102, 140401 (2009).

\section{Acknowledgements}

This work was supported in part by the National Science Foundation. We are indebted to N. Mehta and S. Rittenhouse for extensive discussions and for access to their unpublished derivations before publication. We also thank F. Ferlaino, S. Knoop, H.-C. Nägerl and R. Grimm from the Innsbruck group for discussions about their experimental data.

\section{Author contributions}

The authors contributed equally to the manuscript.

\section{Additional information}

Supplementary information accompanies this paper on www.nature.com/naturephysics. Reprints and permissions information is available online at http://npg.nature.com/ reprintsandpermissions. Correspondence and requests for materials should be addressed to C.H.G. 